\title{
NONEXTENDED QUADRATIC FORMS OVER POLYNOMIAL RINGS OVER POWER SERIES RINGS
}

\author{
RAMAN PARIMALA
}

\begin{abstract}
If $\boldsymbol{R}$ is a complete discrete valuation ring, then every quadratic space over $R[T]$ is extended from $R$. We here show by an example that a corresponding result for higher-dimensional complete regular local rings is not valid.
\end{abstract}

It was proved in [3] that if $R$ is any complete discrete valuation ring, every quadratic space over $R[T]$ is extended from $R$. We show in this note that if $R=\mathbf{R}[[X, Y]]$, there exist anisotropic quadratic spaces over $R[T]$ which are not extended from $R$. This is in contrast to a result of Mohan Kumar and Lindel in the linear case [2, Theorem 5.1, p. 150].

Let $R=\mathbf{R}[[X, Y]], R$ denoting the field of real numbers. Let $\mathbf{H}$ be the quaternions over $\mathbf{R}$ and $A=\mathbf{H}[[X, Y]]$ the ring of formal power series in the variables $X$ and $Y$ over $\mathbf{H}$. We have an $A[T]$-linear map $A[T]^{2} \stackrel{\eta}{\rightarrow} A[T]$ defined by $(1,0) \rightarrow X T$ $+i,(0,1) \rightarrow Y T+j$ which is clearly a surjection. Let $P$ be the kernel of $\eta$.

Proposition. The module $P$ is nonfree projective over $A[T]$.

PROof. The first projection of $A[T]^{2}$ onto $A[T]$ maps $P$ isomorphically onto the left ideal $\mathfrak{A}$ of $A[T]$ generated by $1+Y^{2} T^{2}$ and $1+(i X+j Y) T-k X Y T^{2}[4, \mathrm{p}$. 143]. We prove that $P$ is not free by showing that $\mathfrak{A}$ is not principal. We note first that $\mathfrak{A}$ is not the unit ideal since it is generated modulo $X$ by $1+j Y T$ which is not a unit in $\mathbf{H}[[Y]][T]$. Suppose $\mathfrak{A}$ is principal, generated by $f$. Then $\operatorname{deg}_{T} f<2$. If $\operatorname{deg}_{T} f=0$, then it follows that $\mathfrak{A}$ is the unit ideal, which is not the case. If $\operatorname{deg}_{T} f=2$, then $1+Y^{2} T^{2}$ and $1+(i X+j Y) T-k X Y T^{2}$ are unit left multiples of each other, which is again not possible. Let $\operatorname{deg}_{T} f=1$ and $f=a+b T$, $a, b \in \mathbf{H}[[X, Y]]$. Then $a$ is a unit and we assume $a=1$ so that $f=1+b T$, $b \in \mathbf{H}[[X, Y]]$. We then have

$$
\begin{gathered}
1+Y^{2} T^{2}=(1+c T)(1+b T), \\
1+(i X+j Y) T-k X Y T^{2}=(1+d T)(1+b T) .
\end{gathered}
$$

From the first equation we get $c=-b,-b^{2}=Y^{2}$. This implies that $b=\lambda Y$, $\lambda \in \mathbf{H}[[X, Y]]$. From the second equation we get $d+b=i X+j Y, d b=-k X Y$ so that we have

$$
(i X+j Y) \lambda=-(k X+Y)
$$

Received by the editors September 4, 1980.

AMS (MOS) subject classifications (1970). Primary 15A63, 18 F25.

Key words and phrases. Projective modules, quadratic spaces, extendibility.

() 1981 American Mathematical Society 0002-9939/81/0000-0502/\$01.50 
If $\lambda=\lambda_{0}+\lambda_{1} X+\lambda_{2} Y+\ldots, \lambda_{i} \in \mathbf{H}$, we have $i \lambda_{0}=-k, j \lambda_{0}=-1$, a contradiction, which proves the proposition.

The reduced norm on $P$ [1, Theorem 2.1] gives rise to a quadratic space of rank 4 and discriminant 1 over $R[T]$. This space is anisotropic and not extended from $R$ (in fact indecomposable) in view of [1, Theorem 4.6].

\section{REFERENCES}

1. M. A. Knus, M. Ojanguren and R. Sridharan, Quadratic forms and Azumaya algebras, J. Reine Angew. Math. 303/304 (1978), 231-248.

2. T. Y. Lam, Serre's conjecture, Lecture Notes in Math., vol. 635, Springer-Verlag, Berlin and New York, 1978.

3. Raman Parimala, Quadratic forms over polynomial rings over Dedekind domains, Amer. J. Math. 100 (1978), 913-928.

4. S. Parimala and R. Sridharan, Projective modules over polynomial rings over division rings, J. Math. Kyoto Univ. 15 (1975), 129-148.

Tata Institute of Fundamental Research, Homi Bhabha Road, Bombay 400 005, India 